\title{
A novel option for preoperative endoscopic marking with India ink in totally laparoscopic distal gastrectomy for gastric cancer: A useful technique considering the morphological characteristics of the stomach
}

\author{
TAKAYA TOKUHARA, EIJI NAKATA, TOSHIYUKI TENJO, ISAO KAWAI, SYUNPEI SATOI, \\ KEISUKE INOUE, MARIKO ARAKI, HIROFUMI UEDA and CHIHIRO HIGASHI
}

Department of Surgery, Otori Stomach and Intestines Hospital, Sakai, Osaka 593-8311, Japan

Received September 16, 2016; Accepted February 10, 2017

DOI: 10.3892/mco.2017.1191

\begin{abstract}
In totally laparoscopic distal gastrectomy (TLDG) for gastric cancer, accurately determining the proximal resection line may be difficult. This is because identifying the lesion intracorporeally is impossible, due to the lack of tactile sense, and, in addition, unlike the intestine, the most proximal site of the lesion is often different from the main site due to the distorted shape of the stomach. The aim of this study was to introduce a novel method of preoperative endoscopic marking with India ink, taking into consideration the morphological characteristics of the stomach. Between July, 2013 and April, 2016, 20 patients who underwent TLDG were enrolled in this study. Within the 3 days preceding the operation, after identifying the most proximal site of the lesion on the overlooking image of an endoscope, India ink was injected into the spot on the oral side of this site. The stomach was transected along the proximal border of the marked area. In all cases, the marked sites were localized and clearly identified during the operation, and the proximal resection margins were found to be negative on postoperative pathological examination. The mean length of the proximal margin was $46.0 \pm 14.0 \mathrm{~mm}$. In conclusion, this preoperative endoscopic marking method may be useful in TLDG for gastric cancer.
\end{abstract}

\section{Introduction}

Since laparoscopic-assisted distal gastrectomy (LADG) was reported by Kitano et al in 1994, techniques for laparoscopic distal gastrectomy (LDG) in the treatment of early gastric cancer have been established in several countries (1). Until

Correspondence to: Dr Takaya Tokuhara, Department of Surgery, Otori Stomach and Intestines Hospital, 653-1 Kami, Nishi-ku, Sakai, Osaka 593-8311, Japan

E-mail: sur123@osaka-med.ac.jp

Key words: gastric cancer, totally laparoscopic distal gastrectomy, preoperative endoscopic marking, India ink recently, LADG has been frequently performed; in this procedure, lymph node dissection is laparoscopically performed, but transection of the stomach and anastomosis is performed through an epigastric minilaparotomy. By contrast, in totally laparoscopic distal gastrectomy (TLDG), which does not require a minilaparotomy, all surgical procedures, including lymph node dissection, gastric resection and anastomosis, are performed in a wide laparoscopic visual field. Therefore, TLDG was introduced with Billroth I (B-I) reconstruction or antecolic Roux-en-Y (R-Y) reconstruction in 2013 (2,3). B-I reconstruction was used when the tension in the gastroduodenostomy was low, and R-Y reconstruction was used when the gastric remnant was small (4).

In TLDG for gastric cancer, determining the proximal resection line with adequate margins is often difficult, particularly when the tumor is close to the upper third of the stomach. This is because it is impossible to identify the lesion or clips applied preoperatively in the laparoscopic view due to the lack of tactile sense; in addition, unlike the intestine, the most proximal site of the lesion is often different from the main site due to the distorted shape of the stomach. Several promising techniques for accurately determining the proximal line, such as intraoperative radiography, intraoperative endoscopy and laparoscopic ultrasonography, have been described, but an optimal procedure has yet to be established (5-8).

In this prospective study, a novel method of preoperative endoscopic marking with India ink was applied, taking into consideration the morphological characteristics of the stomach, in order to accurately determine the proximal resection line in TLDG for gastric cancer.

\section{Patients and methods}

Patients. Between July, 2013 and April, 2016, 20 patients who underwent TLDG were enrolled in this study. The diagnosis for all patients was gastric cancer located in the middle third of the stomach. The indication for laparoscopic gastrectomy at our institution (Otori Stomach and Intestines Hospital, Sakai, Japan) is T1N0M0 gastric cancer, according to the Japanese Classification of Gastric Carcinoma (9), that is not a candidate for endoscopic submucosal dissection. The study protocol was 
approved by the Human Ethics Review Committee of the Otori Stomach and Intestines Hospital. Following obtaining written informed consent from the patients, TLDG with preoperative endoscopic marking was performed.

Technique. All the patients underwent gastroendoscopy within the 3 days preceding the operation. After identifying the proximal margin of the lesion, endoscopic metal clips were placed in the vicinity of the proximal margin. To identify which clip was applied at the most proximal site, continuous observation with the overlooking image was performed (Fig. 1). Finally, endoscopic marking with India ink was performed on the oral side of the most proximal clip (Figs. 2-4). As previously reported, $0.1 \mathrm{ml}$ of India ink was injected into the submucosal layer (10). After lymphadenectomy was performed in accordance with the Japanese gastric cancer treatment guidelines (11), the marked site was laparoscopically identified during the operation (Fig. 5). The stomach was transected along the proximal border of the marked area (Figs. 6 and 7). B-I reconstruction was performed when the tension in the gastroduodenostomy was low, whereas R-Y reconstruction was performed when the gastric remnant was small.

\section{Results}

Patient and operative characteristics. The present study included 13 men and 7 women, with a mean age \pm standard deviation (SD) of 66.7 \pm 8.4 years (range $45-78$ years). In all patients, the procedure was successfully performed laparoscopically. B-I reconstruction was performed in 7 patients and R-Y reconstruction was performed in 13 patients. On postoperative pathological examination, 16 patients were stage IA, 3 were stage IB and 1 was stage IIA, according to the Japanese Classification of Gastric Carcinoma (9). The mean duration from the endoscopic marking to the operation was $2.0 \pm 0.76$ days (range, $1-3$ days).

In all cases, the marked sites were localized and clearly identified during the operation; no complications with endoscopic marking using India ink were encountered. On histological examination of the formalin-fixed specimens, the proximal resection margins were negative in all the patients. The mean length $\pm \mathrm{SD}$ of the proximal margin was $46.0 \pm 14.0 \mathrm{~mm}$ (range, $25-77 \mathrm{~mm}$ ).

\section{Discussion}

In TLDG for gastric cancer, proximal gastric resection must be performed intracorporeally in the absence of tactile sense prior to reconstruction. Thus, a technique that may be applied visually is required to accurately determine the proximal resection line. Okamura et al reported that preoperative endoscopic marking with India ink should be performed within 3 days prior to gastrectomy for early gastric cancer and the suitable amount of India ink injected into the submucosal layer was $0.1 \mathrm{ml}$ (10). Therefore, this technique was applied for preoperative endoscopic marking in TLDG. In all cases, the marked sites were localized and clearly identified during the operation; thus, there was no need for intraoperative portable radiography or endoscopy.

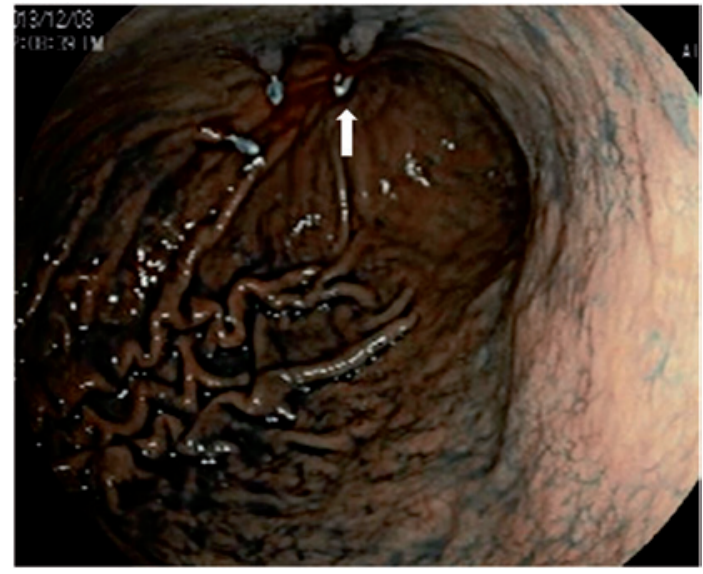

Figure 1. The most proximal clip (arrow) is identified on the overlooking image of an endoscope.

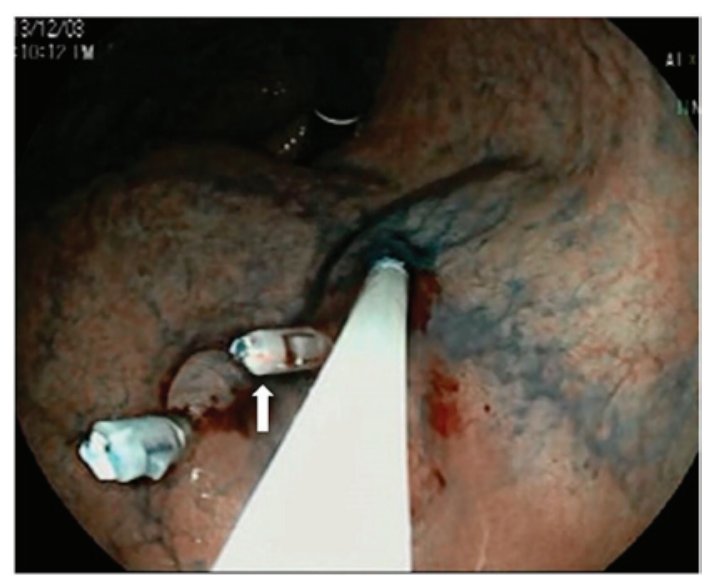

Figure 2. India ink is injected into the spot on the oral side of the most proximal clip (arrow).

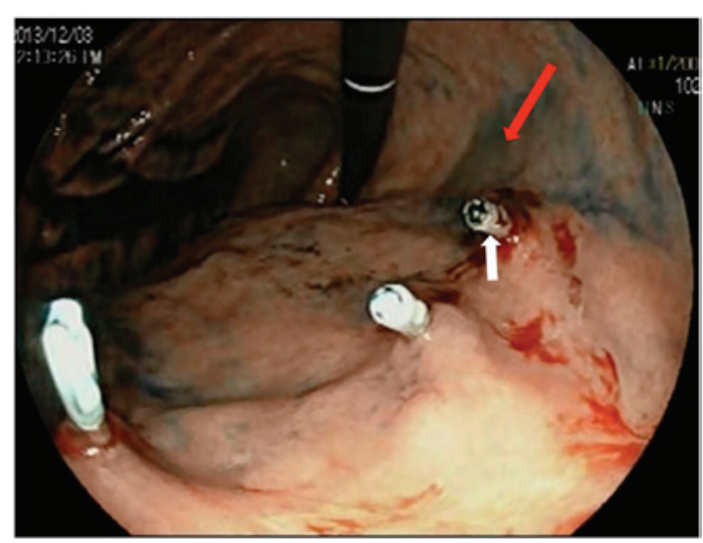

Figure 3. The most proximal clip (white arrow) and India ink (red arrow) on the inverted image of an endoscope.

Unlike the intestine, the stomach is considered to have a distorted shape, as the length of the greater curvature is approximately five times that of the lesser curvature. Therefore, the most proximal site of the gastric cancer is often different from the main site. Taking this morphological characteristic of the stomach into consideration, the most proximal site of the lesion 


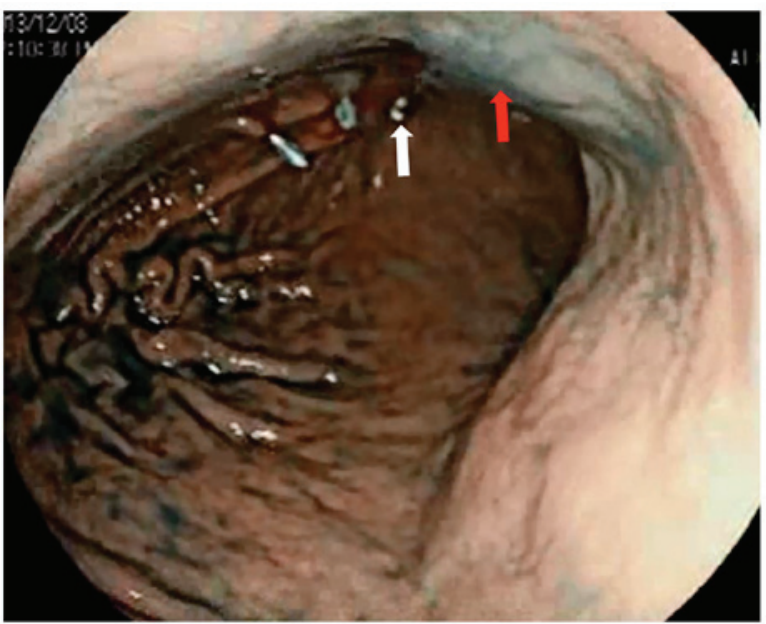

Figure 4. The most proximal clip (white arrow) and India ink (red arrow) on the overlooking image of an endoscope.

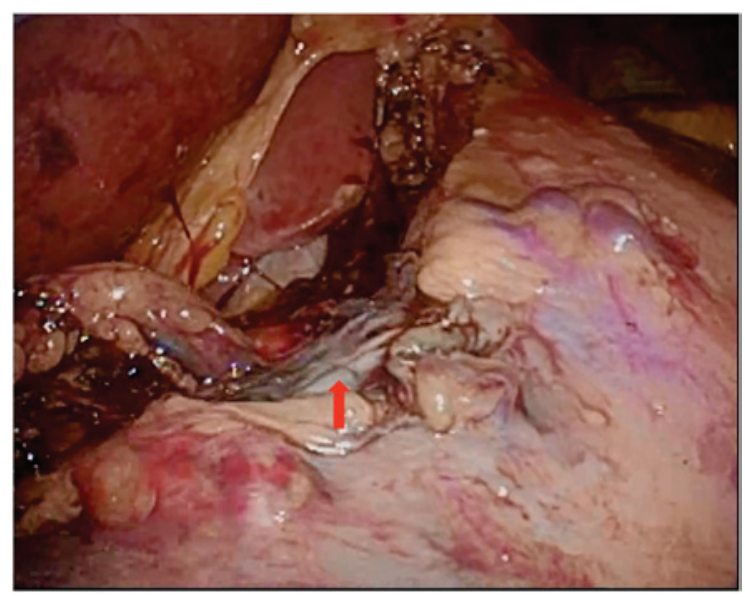

Figure 5. Laparoscopic view of the area marked with India ink (arrow).

must be identified with certainty on the overlooking image during preoperative endoscopy. In addition, India ink must be injected into the spot on the oral side of this site. During the operation, the stomach is transected along the proximal border of the marked area. In the present study, the proximal margins were negative in all cases, and the mean length of the proximal margin was $46.0 \pm 14.0 \mathrm{~mm}$.

Endoscopic clipping was performed in the vicinity of the lesion. This procedure has two aims: To definitively identify the most proximal wedge of the lesion on the overlooking image of an endoscope prior to the injection of India ink, and to make use of an intraoperative portable radiograph or endoscope when the marked site is unclear or too wide during the operation. In all cases, there was no staple dysfunction due to the presence of endoscopic clips, as the stomach was transected along the proximal border of the marked area.

In conclusion, preoperative endoscopic marking with India ink, taking into consideration the morphological characteristics of the stomach, may be a useful technique in TLDG for gastric cancer. However, the number of patients included in this study was small and investigation of a large number of patients is required to draw definitive conclusions on the utility of this marking technique.

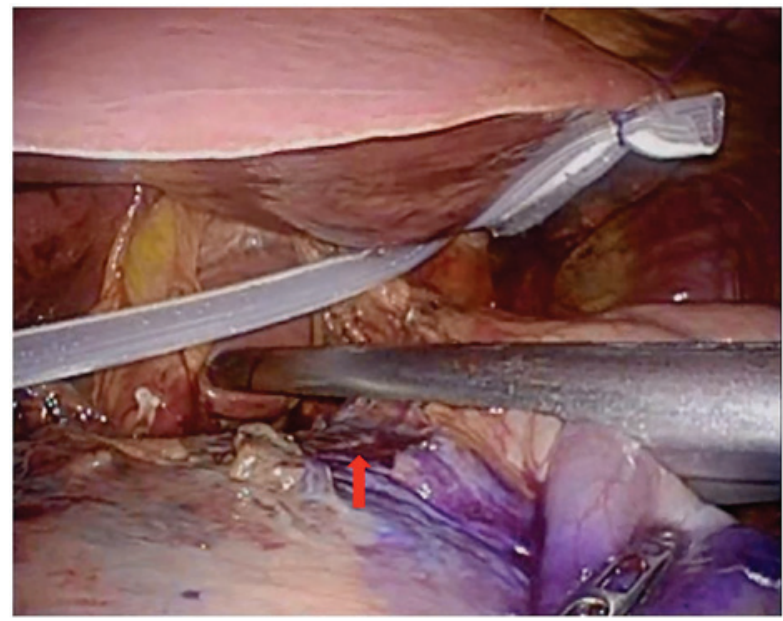

Figure 6. The stomach has been transected along the proximal border of the marked area (arrow).

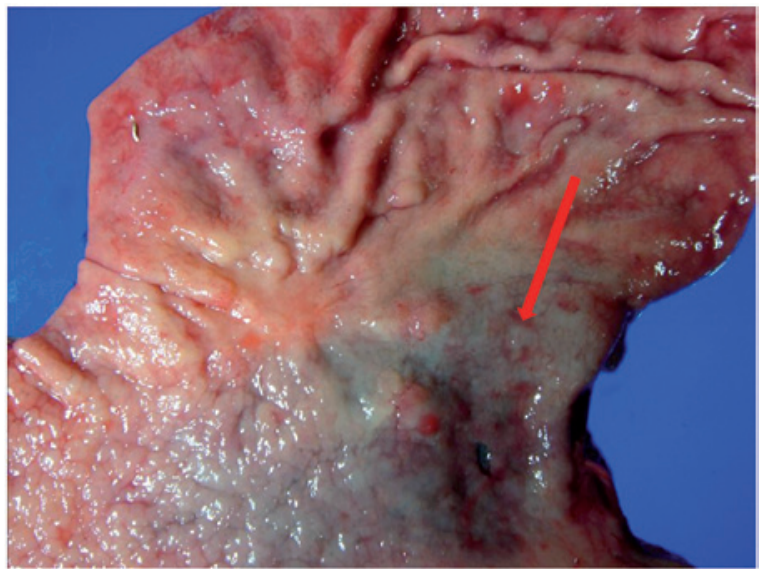

Figure 7. The marked area (arrow) on the mucosal surface of the resected specimen.

\section{Acknowledgements}

The authors would like to thank Dr Nobuhiko Tanigawa, Professor Emeritus of Osaka Medical College, and Dr Kazuhisa Uchiyama, Professor of Osaka Medical College, for providing the idea for this study and for their sincere encouragement.

\section{References}

1. Kitano S, Iso Y, Moriyama M and Sugimachi K: Laparoscopy-assisted Billroth I gastrectomy. Surg Laparosc Endosc 4: 146-148, 1994.

2. Kanaya S, Gomi T, Momoi H, Tamaki N, Isobe H, Katayama T, Wada Y and Ohtoshi M: Delta-shaped anastomosis in totally laparoscopic Billroth-I gastrectomy: New technique of intraabdominal gastroduodenostomy. J Am Coll Surg 195: 284-287, 2002.

3. Takaori K, Nomura E, Mabuchi H, Lee SW, Agui T, Miyamoto Y, Iwamoto $\mathrm{M}$, Watanabe $\mathrm{H}$ and Tanigawa $\mathrm{N}$ : A secure technique of intracorporeal Roux-Y reconstruction after laparoscopic distal gastrectomy. Am J Surg 189: 178-183, 2005.

4. Lee SW, Tanigawa N, Nomura E, Tokuhara T, Kawai M, Yokoyama K, Hiramatsu M, Okuda J and Uchiyama K: Benefits of intracorporeal gastrointestinal anastomosis following laparoscopic distal gastrectomy. World J Surg Oncol 10: 267, 2012. 
5. Kim HI, Hyung WJ, Lee CR, Lim JS, An JY, Cheong JH, Choi SH and Noh SH: Intraoperative portable abdominal radiograph for tumor localization: A simple and accurate method for laparoscopic gastrectomy. Surg Endosc 25: 958-963, 2011.

6. Kinoshita T, Shibasaki H, Oshiro T, Ooshiro M, Okazumi S and Katoh R: Comparison of laparoscopy-assisted and total laparoscopic Billroth-I gastrectomy for gastric cancer: A report of short term outcomes. Surg Endosc 25: 1395-1401, 2011.

7. Kanaya S, Kawamura Y, Kawada H, Iwasaki H, Gomi T, Satoh S and Uyama I: The delta-shaped anastomosis in laparoscopic distal gastrectomy: Analysis of the initial 100 consecutive procedures of intracorporeal gastroduodenostomy. Gastric Cancer 14: 365-371, 2011.

8. Hyung WJ, Lim JS, Cheong JH, Kim J, Choi SH, Song SY and Noh SH: Intraoperative tumor localization using laparoscopic ultrasonography in laparoscopic-assisted gastrectomy. Surg Endosc 19: 1353-1357, 2005.
9. Japanese Gastric Cancer Association: Japanese classification of gastric carcinoma: III English edition. Gastric Cancer 14: 101-112, 2011.

10. Okamura R, Hamagaki M, Hayashi M, Kuwana M, Yamamoto H, Shinofuji M, Yamamoto A, Inoue S, Satomi T, Fujita N, et al: Preoperative endoscopic injection of carbon ink as a method to demarcate area for gastrectomy applied to early gastric cancers. Stomach and Intestine 23: 441-445, 1988

11. Japanese Gastric Cancer Association: Japanese gastric cancer treatment guidelines 2010 (ver. 3). Gastric Cancer 14: 113-123, 2011. 